\title{
Transillumination imaging through scattering media by use of photorefractive polymers
}

\author{
D. D. Steele, B. L. Volodin, O. Savina, B. Kippelen, and N. Peyghambarian \\ Optical Sciences Center, The University of Arizona, Tucson, Arizona 85721
}

H. Röckel

Beckman Institute, California Institute of Technology, Pasadena, California 91125

\begin{abstract}
S. R. Marder
Beckman Institute and Jet Propulsion Laboratory, California Institute of Technology, Pasadena, California 91109
\end{abstract}

Received May 16, 1997

\begin{abstract}
We demonstrate the use of a near-infrared-sensitive photorefractive polymer with high efficiency for imaging through scattering media, using an all-optical holographic time gate. Imaging through nine scattering mean free paths is performed at $800 \mathrm{~nm}$ with a mode-locked continuous-wave Ti:sapphire laser. (c) 1998 Optical Society of America

OCIS codes: $190.5330,160.5470,160.5320,110.3080$.
\end{abstract}

Imaging through highly scattering media by use of optical radiation has received particular attention recently because of its potential applications in medical diagnostics. More-traditional medical imaging techniques, such as computed tomography, magnetic resonance imaging, and ultrasound, ${ }^{1}$ are capable of measuring different physical quantities, and each has its limitations. Adding imaging with optical radiation to this list would provide a complementary and valuable tool for diagnostics. With optical radiation one can avoid the hazards of ionizing radiation while obtaining potentially diffraction-limited images, which offer a resolution advantage compared with clinical ultrasound systems, ${ }^{2}$ and spectroscopic techniques may discern functional information. Although it is not strongly absorbed, optical radiation is heavily scattered in biological tissue by refractive-index inhomogeneities. As a result, transillumination of tissue produces light that has experienced no scattering events (ballistic light), weakly scattered light that emerges almost collinearly with the incident radiation (snake light), and highly scattered (diffuse) light. $^{3} \quad$ Ballistic and snake light provide useful information about absorption centers in the medium for shadowgram imaging. Conversely, diffuse light can emerge from the medium in potentially any direction and usually at times later than the ballistic and snake light. To use diffuse light for imaging one is required to model photon transport and to employ algorithms to reconstruct the image. ${ }^{4}$ So the main challenge when one is forming shadowgram images through scattering media is to extract the information-bearing light selectively without imaging the diffuse light. ${ }^{3}$

A variety of methods have been developed to extract the ballistic light from the background of scattered light. Among these are spatial filtering techniques ${ }^{5}$ and incoherent ${ }^{6,7}$ and coherent ${ }^{8,9}$ methods that take advantage of the temporal stretching of a short pulse of light in a scattering medium.
In this Letter we demonstrate the use of photorefractive (PR) polymers as the recording medium for timegated holographic imaging through scattering media. This method offers fast acquisition time because an entire two-dimensional image can be acquired at one time, as opposed to the point-by-point scanning of certain techniques. ${ }^{5,10}$ Furthermore, this approach is straightforward to implement and does not involve any computations.

In this method a hologram of an object embedded in a scattering medium is formed in a PR polymer by the temporal overlap of a reference pulse and the ballistic light from a stretched image-bearing pulse. The filtering of ballistic from scattered light is thus achieved without any digital processing. This hologram can then be reconstructed in real time by diffraction of a probe beam.

PR holographic time gating was demonstrated previously in inorganic crystals, such as $\mathrm{BaTiO}_{3},{ }^{11}$ and has important advantages associated with real-time erasable recording. Inorganic PR crystals, though, are generally rather expensive, limited in size, and can exhibit beam fanning effects that are a source of noise in imaging, whereas PR polymers offer the benefits of low cost, the ability to form large-area films, high reconstruction efficiency in relatively thin layers, high angular bandwidth, and structural flexibility.

The details of PR polymer design, properties, and performance have already been described. ${ }^{12}$ Recently we designed a PR polymer that exhibits high performance from 700 to $900 \mathrm{~nm},{ }^{13}$ which is desirable for medical imaging because this region of transparency is one of the best for biological tissue and is covered by commercially available Ti:sapphire lasers and by efficient and inexpensive laser diodes. The polymer used was a composite of the dye $2, N, N$-dihexylamino-7-dicyanomethylidenyl-3, 4, 5, 6, 10-pentahydronaphthalene (25 wt. \%), the polymer matrix poly(vinylcarbazole) $: N$ - ethylcarbazole (48:25 wt. \%), 
and the photosensitizer (2, 4, 7-trinitro-9-fluorenylidene)malononitrile ( $2 \mathrm{wt} . \%$ ). The $105-\mu \mathrm{m}$-thick polymer layer was sandwiched between two 1-mm-thick glass slides coated with transparent indium tin oxide electrodes.

The experimental setup can be seen in Fig. 1. We used a Ti:sapphire laser operating at $800 \mathrm{~nm}$ with a pulse duration of $\sim 150 \mathrm{fs}$ (full width at half-maximum), a repetition rate of $\sim 90 \mathrm{MHz}$, and an average output power of $\sim 250 \mathrm{~mW}$. The imaging was performed in a degenerate four-wave mixing geometry with the two writing beams subtending an angle of $28^{\circ}$ (Fig. 1, referenced and object arms) and interfering in the polymer to generate a hologram by the PR process. The polymer sample was tilted $55^{\circ}$ with respect to the bisector of the two writing beams and was subject to an applied voltage of $5.5 \mathrm{kV}$. The hologram was reconstructed by the probe beam, which was counterpropagating with the reference beam. For best efficiency the writing beams were $s$ polarized and the reading beam was $p$ polarized. ${ }^{12}$ The fact that the reconstruction was done in real time points to the potential to create in vivo dynamic images of biological tissue based on further optimization of near-infrared-sensitive PR polymers.

To demonstrate the temporal stretching of a short pulse on propagation through a scattering medium we measured the cross correlation of a stretched pulse with an unstretched reference pulse by frequency upconversion. Figure 2 shows the cross correlation with a pulse after it has passed through $10 \mathrm{~mm}$ of water (dotted curve) and with a pulse that has traversed $10 \mathrm{~mm}$ of a weakly scattering medium (highly dilute milk). As seen here, even a weakly scattering medium [fewer than three scattering mean free paths (MFP's)] leads to considerable stretching of the pulse, illustrating the effectiveness of the holographic time-gating method.

To determine the scattering cross section empirically for different media we performed ballistic light attenuation measurements, using calibrated polystyrene spheres (Polysciences, Inc.). The precise sizes and shapes of the spheres permitted accurate comparison with Mie theory. We measured attenuation by monitoring the light power after the light had passed through the scattering medium and two $6-\mathrm{mm}$ apertures separated by $910 \mathrm{~mm}$ (0.14-msr solid angle). The inset of Fig. 2 shows measured curves of ballistic light attenuation through $10-\mathrm{mm}$ water suspensions of various concentrations and diameters of microspheres. For this experiment we used an effective scattering coefficient $\mu_{s}$, defined as $I / I_{0}=\exp \left(-\mu_{s} d\right)$, where $I$ is the irradiance measured after the scattering medium, $I_{0}$ is the irradiance measured after a reference cuvette filled with water, and $d$ is the thickness of the scattering medium. Because polystyrene does not absorb at this wavelength, the total light attenuation was due solely to scattering. A linear fit of these data for the $1.072-\mu \mathrm{m}$ spheres gives an effective scattering cross section $\sigma_{s}$ of $1.4 \times 10^{-8} \mathrm{~cm}^{2}$, exactly the value calculated by Hee et al. ${ }^{10}$ This cross section is related to $\mu_{s}$ as $N \sigma_{s}=\mu_{s}$, where $N$ is the number density of scattering particles. The number of MFP's traversed is $\mathrm{MFP}=\mu_{s} d$.
The test object used in the imaging experiments was a standard U.S. Air Force resolution target positioned in front of the scattering cell. To illustrate the influence of the size of the particles of the imaging results, calibrated polystyrene spheres of three diameters, $0.548,1.072$, and $2.836 \mu \mathrm{m}$, were employed. The cuvettes used were either 1 or $10 \mathrm{~mm}$ thick. In all cases similar results were obtained, with the most important factor being the total ballistic light attenuation. For this reason we show only the results acquired with water suspensions of $0.548-\mu \mathrm{m}$ spheres in the $10-\mathrm{mm}$ cuvette.

Figure 3 shows the time-gated image recorded with a mode-locked Ti:sapphire laser in the PR polymer after the light has passed through $10 \mathrm{~mm}$ of a 0.06 -vol. \% microsphere suspension, which corresponds to an effective optical density of $\sim 4.0$, or 9 MFP's, as determined

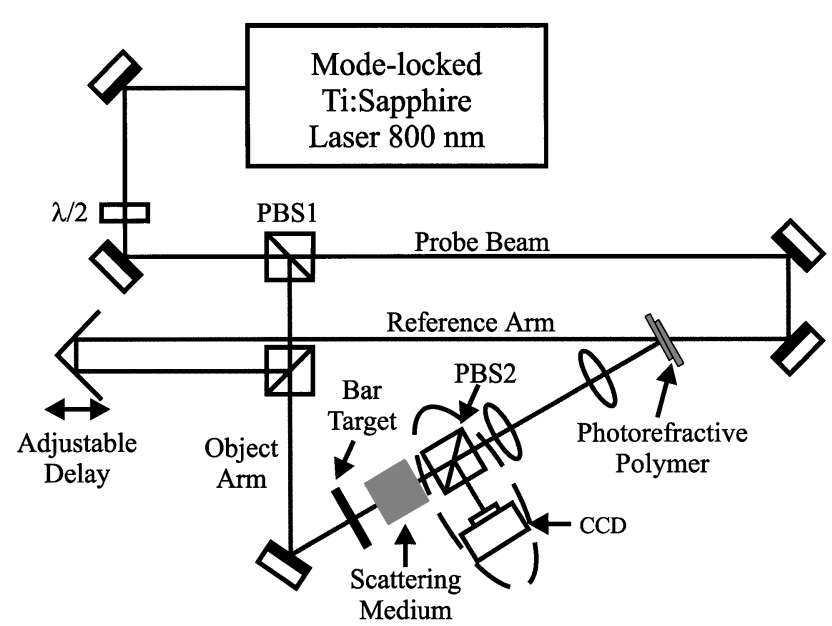

Fig. 1. Experimental setup. The oval area described by the long-dashed curve is actually out of the plane of the setup. The power ratio between the probe and the writing beams was controlled by half-wave plate $\lambda / 2$ and polarizing beam splitter PBS1. A second polarizing beam splitter, PBS2, directed the diffracted beam to the CCD camera (Sony Model XC-73).

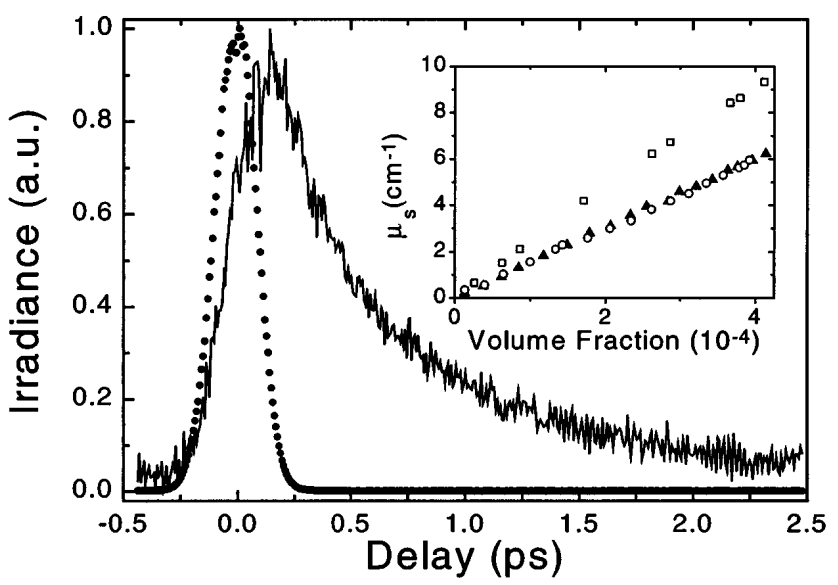

Fig. 2. Cross correlations of an unstretched pulse (dotted curve) and a pulse stretched during propagation through a weakly scattering medium ( $<3$ MFP's). The inset shows the scattering coefficient as a function of volume fractions of 0.548 (filled triangles), 1.072 (squares), and $2.836-\mu \mathrm{m}$ (circles) polystyrene spheres suspended in water. 


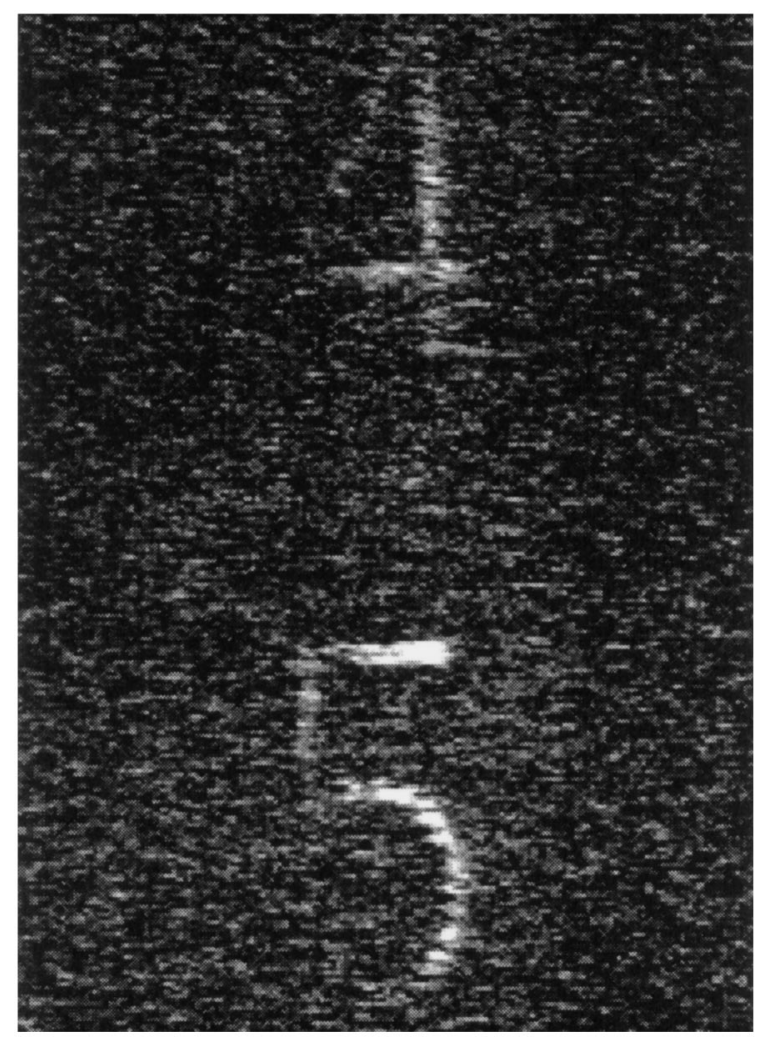

Fig. 3. Time-gated image of the resolution target after the light has passed through $1 \mathrm{~cm}$ of a $0.06 \%$ water suspension of $0.548-\mu \mathrm{m}$ polystyrene spheres.

from the optical attenuation measurements described above. For comparison, $1 \mathrm{~mm}$ of whole milk has an effective optical density of $\sim 5.0$.

Because of the short coherence length of the Ti:sapphire laser in cw operation, it was also possible to perform filtering of the scattered light while running the laser $\mathrm{cw}$. Similar results were obtained with $\mathrm{cw}$ radiation. Ultimately, using $\mathrm{cw}$ light is an advantage because it opens up the possibility of using short-coherence-length laser diodes, allowing one to drastically reduce the cost of a practical system based on this technique.

Holographic imaging with PR polymers offers a number of advantages over other imaging techniques. In addition to the material advantages mentioned above, diffraction efficiency achievable with $100-\mu \mathrm{m}$ thick PR polymer films is comparable with or exceeds what is typically achieved with near-infrared-sensitive, several-millimeter-thick rhodium-doped $\mathrm{PR} \mathrm{BaTiO}_{3}$ crystals. The use of thin layers is particularly important for this application because the angular bandwidth of the imaging system is inversely proportional to the interaction length, and a larger angular bandwidth leads to higher resolution. Moreover, the response time of the polymers $(\sim 1 \mathrm{~s})$ is considerably faster than that of crystals $(\sim 10 \mathrm{~s})$. PR polymers also possess structural flexibility that can lead to significantly improved efficiency by means of molecular engineering. Altogether, these properties grant PR polymers greater technological potential than their inorganic counterparts.

In conclusion, for the first time to our knowledge PR polymers sensitive in the near infrared have been applied to transillumination imaging through scattering media. Imaging through 9 MFP's has been demonstrated by use of 150 -fs pulses as well as of shortcoherence-length $\mathrm{cw}$ radiation.

This research was supported by the U.S. Office of Naval Research through the Multidisciplinary University Research Initiative, Center for Advanced Multifunctional Nonlinear Optical Polymers and Molecular Assemblies, by the U.S. Air Force Office of Scientific Research, and by the National Science Foundation. We thank P. M. W. French for helpful discussions. H. Rockel thanks the Alexander von Humbolt Foundation for a Feodor Lyman postdoctoral fellowship.

\section{References}

1. Z.-H. Cho, J. P. Jones, and M. Singh, Foundations of Medical Imaging (Wiley, New York, 1993).

2. W. R. Hedrick, D. L. Hykes, and D. E. Starchman, Ultrasound Physics and Instrumentation (Mosby, St. Louis, Mo., 1995).

3. S. K. Gayen and R. R. Alfano, Opt. Photon. News 7(3), 16 (1996).

4. See, for example, J. Singer, F. Grunbaum, P. Kohn, and J. Zubelli, Science 248, 990 (1990).

5. See, for example, J. B. Pawley, ed., Handbook of Biological Confocal Microscopy (Plenum, New York, 1990).

6. M. A. Duguay and A. T. Mattick, Appl. Opt. 10, 2162 (1971).

7. L. Wang, P. P. Ho, X. Liang, H. Dai, and R. R. Alfano, Opt. Lett. 18, 241 (1993).

8. N. H. Abramson and K. G. Spears, Appl. Opt. 28, 1834 (1989).

9. E. Leith, C. Chen, H. Chen, D. Dilworth, J. Lopez, J. Lopez, J. Rudd, P.-C. Sun, J. Valdmanis, and G. Vossler, J. Opt. Soc. Am. A 9, 1148 (1992).

10. M. R. Hee, J. A. Izatt, J. M. Jacobson, J. G. Fujimoto, and E. A. Swanson, Opt. Lett. 18, 950 (1993).

11. S. C. W. Hyde, N. P. Barry, R. Jones, J. C. Dainty, P. M. W. French, M. B. Klein, and B. A. Wechsler, Opt. Lett. 20, 1331 (1995).

12. K. Meerholz, B. L. Volodin, Sandalphon, B. Kippelen, and N. Peyghambarian, Nature (London) 371, 497 (1994).

13. B. Kippelen, S. R. Marder, E. Hendrickx, J. L. Maldonado, G. Guillemet, B. L. Volodin, D. D. Steele, Y. Enami, Sandalphon, Y. J. Yao, J. F. Wang, H. Röckel, L. Erskine, and N. Peyghambarian, "Infrared photorefractive polymers and their applications for imaging," Science (to be published). 